\title{
Resolución de Conflictos
}

\section{Autor: David Pérez García}

Afiliación Alumnado de la asignatura "La educación en valores y la práctica de actividad física en colectivos de desigualdad. Grado de Ciencias de la Actividad Física y el Deporte. Universidad de Huelva. 


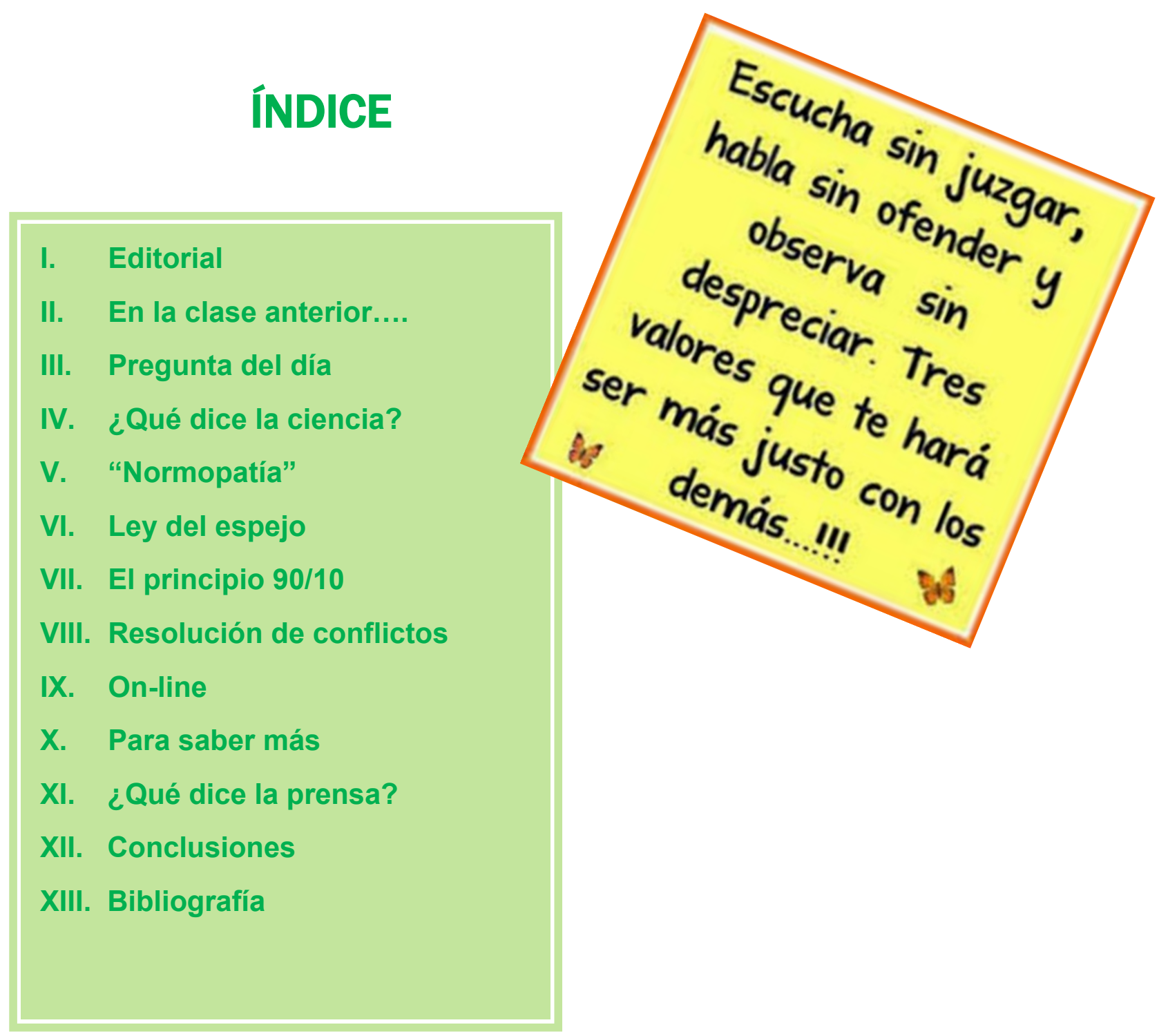

Para reflexionar...

$$
\begin{aligned}
& \text { "iEL CONFLICTO ES UNA } \\
& \text { OPORTUNIDAD DE } \\
& \text { APRENDIZAJE!" }
\end{aligned}
$$

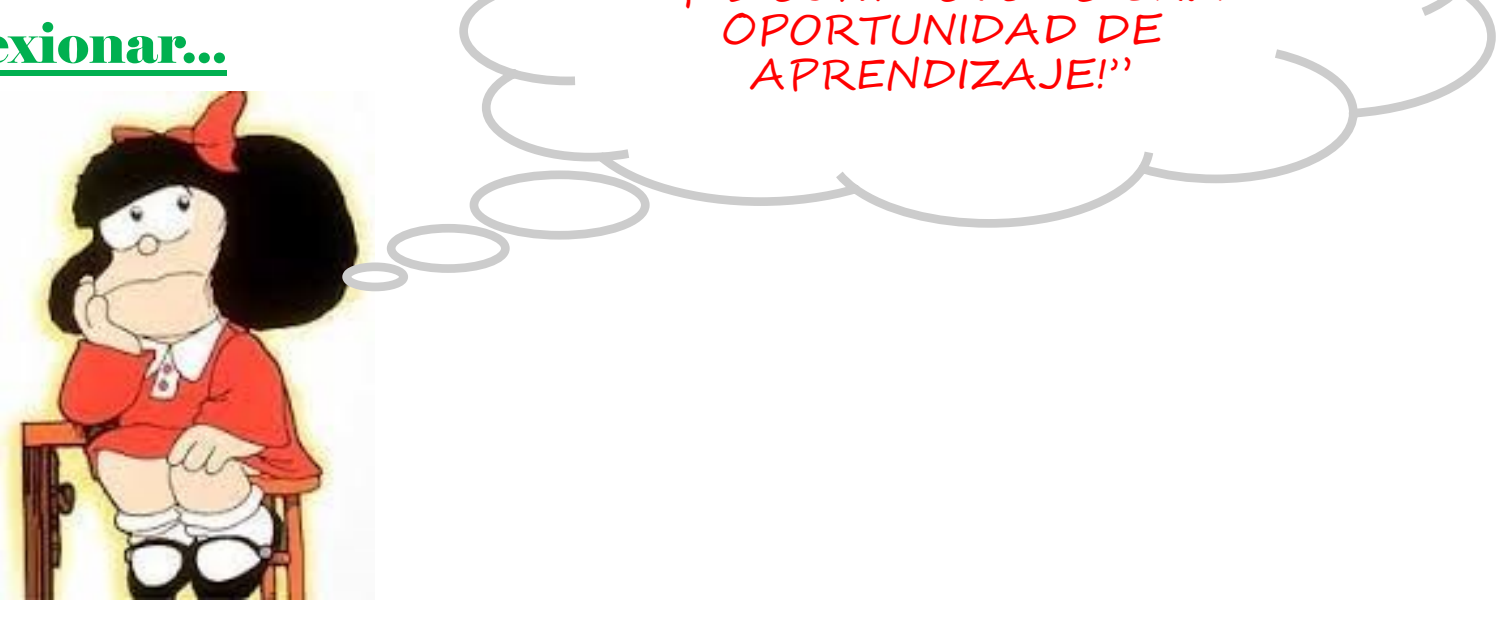




\section{Editorial}

Los conflictos son parte de nuestra vida y una gran fuente de aprendizaje. Tener conflictos significa estar vivo, y lo saludable es aprender a manejarlos.

Por ello, la resolución de conflictos debe tomar un papel crucial en la educación, ya que no tratar las emociones y resolución de conflictos en la enseñanza obligatoria, significa mantener al alumnado en un analfabetismo emocional que les dificultará la superación de los conflictos que se les presenten a todas las edades.
En este boletín abordaremos este tema desde los diferentes contenidos tratados en clase. Además, adjuntaremos una serie de artículos relacionados con la resolución de conflictos, algunos facilitados por compañeros de clase, y otros investigados por los propios autores del boletín. Por otro parte, explicaremos algunos fundamentos más teóricos que representan aspectos muy importantes de la resolución de problemas como por ejemplo la "normopatía", ley del espejo y teoría 90/10. Por último, apoyaremos todos estos contenidos con ejemplos más prácticos como por ejemplo vídeos, actividades, libros y hechos reales.

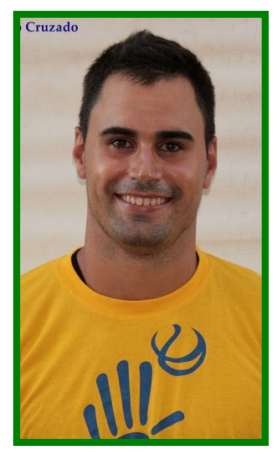

Autor: Sergio Cruzado López.

Sáenz-López,

P. (2014).

Apuntes inéditos de la asignatura "La educación en valores y la práctica de actividad física en colectivos en situa-

\section{Para Recordar}

La clase anterior tuvo lugar el día 26 de Noviembre de 2014, durante la cual se trató principalmente el tema de "SUBCONSCIENTE". En primer lugar, en la clase realizamos una actividad por parejas, en la cual debíamos elegir una emoción (miedo, ira, vergüenza, etc.) y reflexionar sobre ella. De esta manera pudimos observar que las emociones negativas pueden transformarse y tener un carácter positivo. Como conclusiones de esta actividad observamos que las emociones se deben afrontar desde la raíz y que la "culpa" es la emoción en la que más complicado resulta sacar el aspecto positivo.

En segundo lugar, se mencionó que la "autoestima" es la mejor medicina para solucionar y resolver algún problema, aspecto bastante relacionado con el tema principal trabajado en este boletín. En cuanto a los artículos de dicha clase, destacar que se expusieron un total de tres, el primero de ellos llamado, Papel Fundamental del Educador, en el que Alejandro resaltó como conclusión principal la importancia de poner objetivos alcanzables a los alumnos. El segundo de ellos, expuesto por Víctor, trató sobre la autoestima y auto concepto y nos destacó que se obtenían mejores resultados cuando el individuo practicaba actividad física. Por último, Antonio González Crespo, habló sobre como conocernos nosotros mismos nos ayudaba a entender cómo se sentían los demás.

Para terminar, nos gustaría destacar de la clase anterior, la charla que preparó Florencio sobre el Inconsciente y Subconsciente. En dicha charla hubo un aspecto que nos llamó bastante la atención, este fue el hecho de que el inconsciente funcionara en nuestro cerebro como un piloto automático.
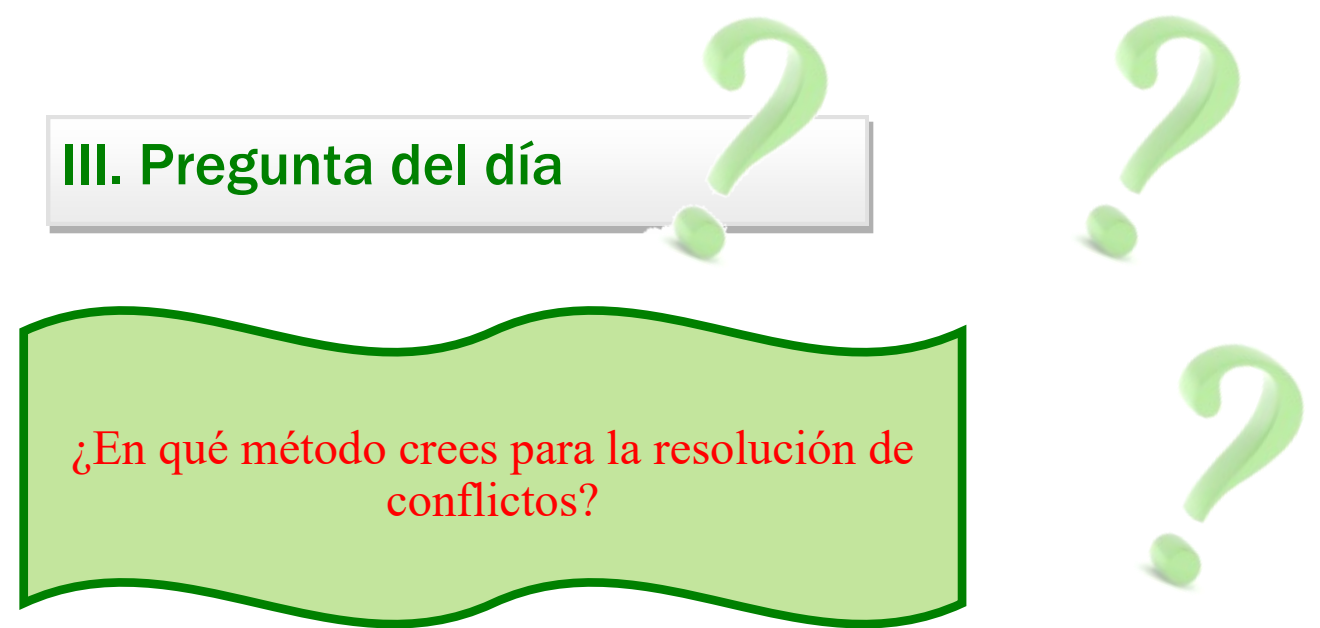
Autor: Antonio Quintero Cano.

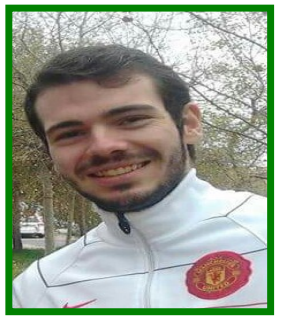

\section{IV. ¿Qué dice la ciencia?}

Título: El proyecto esfuerzo: Un modelo para el desarrollo de la responsabilidad personal y social a través del deporte.

Ruiz, L. M; Rodríguez, P; Martinek, T; Schilling, T; Durán, L. J; Jiménez, P. (2005). El proyecto Esfuerzo: Un modelo para el desarrollo de la responsabilidad personal y social a través del deporte. Revista de educación 341.

El artículo expuesto por el alumno Antonio Quintero hablaba sobre un programa de intervención realizado en la Universidad de Carolina del Norte que fue desarrollado con el fin de ofrecer a niños escolares de entre 8 y 14 años, en su mayoría procedentes de fuera del país y todos de color, provenientes de zonas pobres de la ciudad un programa deportivo extraescolar, y para formar un grupo de mentores en la misma escuela, a la par que para desarrollar la fuerza y resistencia de estos jóvenes socialmente desfavorecidos. Con ello se pretende que dichos niños puedan interactuar entre ellos, hacer amigos y divertirse mientras hacen deporte, y sobre todo ayudar a estos jóvenes a alejarse de los conflictos de su vida cotidiana.

Título: El modelo de enseñanza de la responsabilidad personal y social.

Hellison, D. (1978). El Modelo de Enseñanza de la Responsabilidad Personal y Social a través del Deporte. Revista de Educación 341.

El artículo expuesto por el compañero Jesús Samuel Prieto, trató sobre El Modelo de Enseñanza de la Respon- sabilidad Personal y Social. (Hellison, 1978) el cual asocia dos valores al bienestar y al desarrollo personal: esfuerzo y autogestión, y otros dos valores al desarrollo y la integración social: respeto a los sentimientos y derechos de los demás, y escuchar y ponerse en el lugar de otro para así evitar conflictos. Para conseguir esto, los alumnos debían aprender por niveles comportamientos de responsabilidad personal y social.

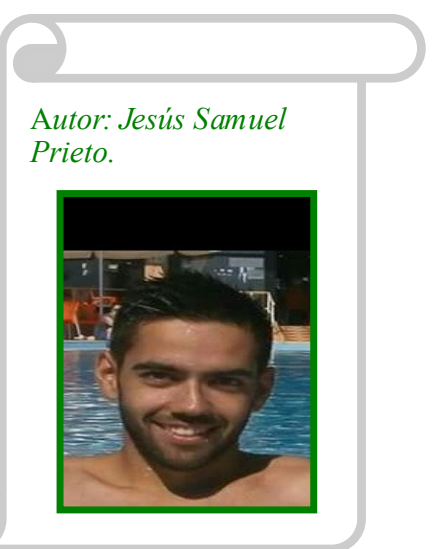

Normopatía. (30 de Mayo 2012). Cyclopaedia. Revisado el 2 de Diciembre de 2014 en, http:// es.wikipedia.org/wiki/Normopat\% C3\%ADa

En psicodinámica, la normopatía designa la tendencia a conformarse excesivamente con las normas sociales del comportamiento sin atreverse a expresar la subjetividad propia, es decir, la normopatía es actuar según las normas, vestirnos como nos indica la moda, hablar como todo el mundo, actuar como todo el mundo, etc.

\section{V. "Normopatía"}

pasivamente lo que la televisión les señala como 'bueno', 'justo' y 'correcto' sin animarse a cuestionar o pensar algo diferente; Condenan a quien lo hace o aceptan que los condenen.

Por último, destacar el hecho de que todos tenemos algo de "normopatía", es decir, todos vemos el mundo desde nuestra normalidad.
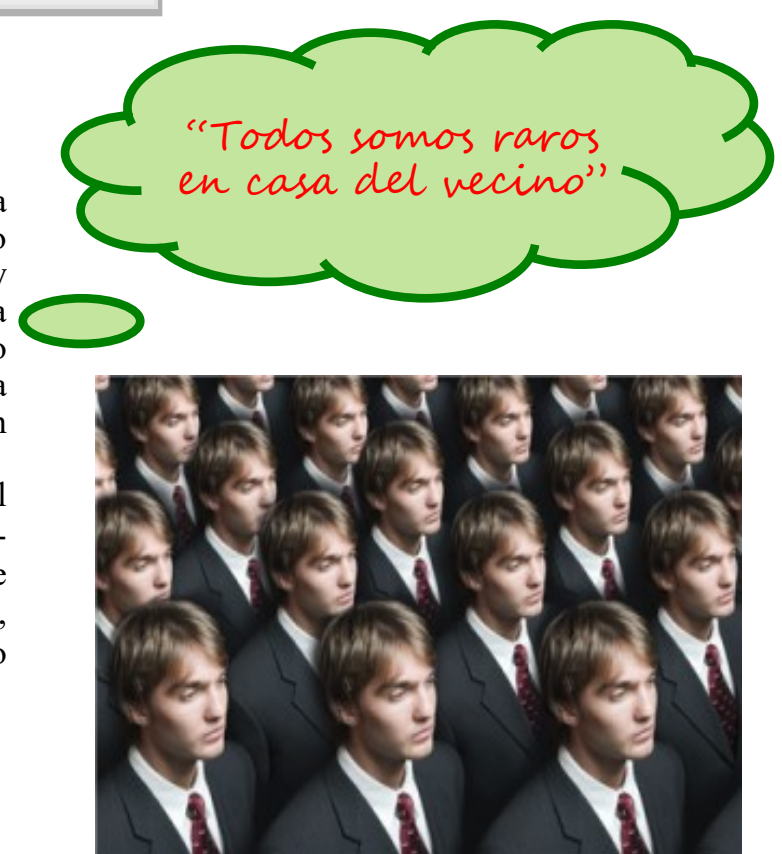
JUZGAR A UNA PERSONA NO DEFINE OUIEN ES ELLA. DEFINE QUIEN ERES TU DEFINE OUIEN ERES TU

\section{Ley del espejo}

- Descubre la Ley del Espejo para ser Feliz. Mejor con salud. Revisado el 2 de Diciembre de 2014, en http:/ mejorconsalud.com/descubre-la-ley-del-espejo-para-serfelizl

- Sáenz-López, P. (2014). Apuntes inéditos de la asignatura "La educación en valores y la práctica de actividad física en colectivos en situación de desigualdad”. Grado de Ciencias del Deporte. Universidad de Huelva.

La ley del espejo nos dice que lo que vemos en los demás es en realidad un reflejo de lo que tenemos dentro nosotros mismos. Así pues, cuando nos molestan ciertos defectos o comportamientos de los demás, podemos deducir con toda seguridad que esos son los nuestros, aunque estén retenidos o reprimidos subconscientemente. Es más, cuanto más nos desagradan es porque más nos duelen los propios.

Por ejemplo, si nos sentimos ofendidos por algo que nos han dicho, eso es porque también nosotros estamos ofen- diendo a alguien, tal vez no a esa persona pero sí a otra. Y si aprendemos a darnos cuenta, y sobre todo a evitarlo, posiblemente también dejaremos de sentirnos ofendidos nosotros.

¿Qué hacemos con lo que sucede sin buscarlo? ¿Y qué hacemos con lo que buscamos?

Es curioso cómo no todas las personas reaccionamos igual ante la misma situación. Porque no todas tenemos lo mismo en el corazón.

En relación con el tema que estamos tratando, la resolución de conflictos, diremos que resulta bastante útil la ley del espejo, ya que nos ayuda a conocernos mejor.

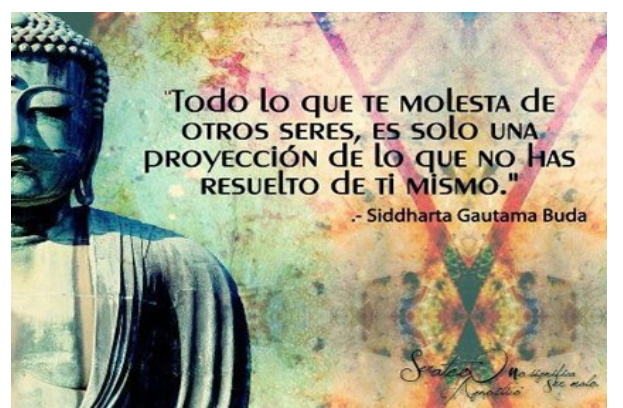

\section{EJERCICIO}

Hacer una lista con todas las cosas que nos molestan de las personas que nos rodean.

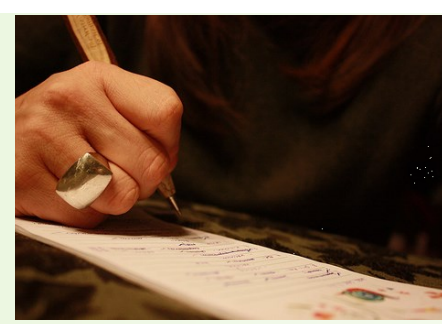

\section{El principio 90/10}

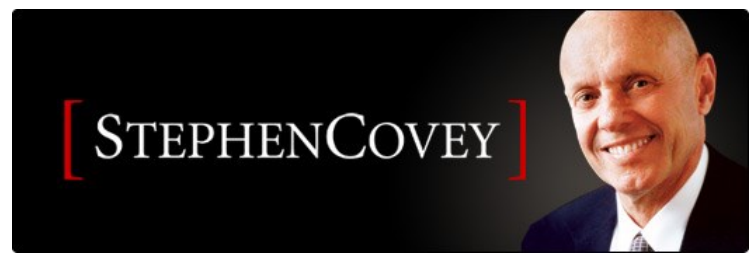

El Principio 90 10, cuyo autor es Stephen Covey, nos dice que una pequeña parte de lo que sucede en la vida diaria es capaz de crear una gran perturbación emocional. Establece que el 10\% de la vida está relacionado con lo que nos pasa, situaciones que nos suceden en la vida y que no se encuentran bajo nuestro control. El restante $90 \%$ está determinado por la forma en que reaccionas a eso que pasa, es decir, lo determinamos nosotros con nuestra reacción.

Usemos un ejemplo... Estás desayunando con tu hija, que tira la taza de café y salpica tu camiseta de trabajo. Tú no tienes control sobre lo que acaba de pasar. Pero lo siguiente que suceda estará determinado por tu reacción. Ante un mismo escenario, podemos reaccionar de diferentes maneras, bien regañando a nuestra hija y alterándonos, lo que seguramente desencadenará una consecución de sucesos negativos, como puede ser que al estar alterado se te olvide un portafolio que tenías que llevar al trabajo, lo que te enojará aún más, etc. Así el día empezó terrible y se pondrá cada vez peor, ya que la forma en que reaccionaste esos 5 segundos determinó el cauce de tu mal día. Sin embargo podemos reaccionar diciéndole simplemente que no se preocupe pero que tenga más cuidado la próxima vez, una reacción que nos ayuda a mantener la calma y que todo se desarrolle como es debido.

(Ppt: Slideshare, 2008) 


\section{Resolución de conflictos}

Sáenz-López, P. (2014). Apuntes inéditos de la asignatura "La educación en valores y la práctica de actividad fisica en colectivos en situación de desigualdad”. Grado de Ciencias del Deporte. Universidad de Huelva.

Partiremos de una serie de preguntas que nos harán reflexionar y acercarnos a la base de los conflictos. Estas son:

- ¿Por qué nos enfadamos?

- ¿Qué me molesta que me digan? ¿Cómo reaccionamos?

Existe un comportamiento humano que es bastante habitual a la hora de afrontar un conflicto, es cual es "o ataco o huyo". Este tipo de comportamiento recibe el nombre de COMPORTAMIENTO REPTILIANO.

Frente a esto, una gran herramienta que ayuda sin duda a evitar conflictos es la educación. Nuestro profesor defiende que la educación es control, y por tanto si aprendemos a controlarnos ante las situaciones que se nos presentan, podremos afrontar de una mejor manera los problemas.

Partiendo de esta base, proponemos los siguientes pasos, a cada cual más importante, para la resolución de conflictos, los cuales son:

\section{Tranquilizarse}

Esto resulta de gran importancia para afrontar el problema determinado de la mejor manera posible y neutralmente. Esto puede facilitar la comunicación, generar confianza y permite además gestionar el proceso de resolución con mayor claridad y serenidad. Con esto se pretende trasmitir calma a las partes implicadas.

\section{Tranquilizar a los afectados}

Es un aspecto fundamental del proceso, ya que tiene mucha influencia en el proceso de resolución, puesto que si se afronta el problema con las partes implicadas aun alteradas, puede desembocar en un agravio de la situación. Esto reducirá las tensiones entre las partes y fomentará la comunicación entre ellos.

\section{Preguntar por los sentimientos}

En este apartado tratamos que las partes implicadas compartan con nosotros la información respecto al conflicto o problema que les concierne y que expresen como les afecta, como se sienten, así como el modo en el que están viviendo la situación.

\section{Resolución colectiva}

Aquí se tratará de facilitará la discusión con la intención de flexibilizar a las partes implicadas, es decir, reducir las causas de la discrepancia y acercar los puntos de vista de ambas partes. El objetivo de este apartado es encontrar una solución consensuada entre dichas partes. Un factor importante en este punto del proceso es no imponer la solución, simplemente se ha de facilitar el acuerdo.

\section{Asumir responsabilidades}

Es aquí cuando las partes implicadas deben empatizar con su opuesto para terminar aceptando el acuerdo. Así se asumirán los diferentes fallos que han tenido durante el conflicto.

\section{Compensar}

Es la culminación del proceso. Una vez que ambas partes han asumido las responsabilidades, debe salir de ellos el compensar a la parte opuesta por los posibles daños causados durante el problema. En caso de que ambas partes no estén dispuestas a compensar según la solución acordada, significa que algo ha fallado en el proceso. Así deberíamos intervenir como mediadores e imponer una solución.

Para terminar, destacaremos un consejo que ofreció Pedro durante la evolución de la clase. Este decía así: No se debe resolver el conflicto de primeras, mientras las partes integrantes están aún "alteradas", como he mencionado anteriormente.
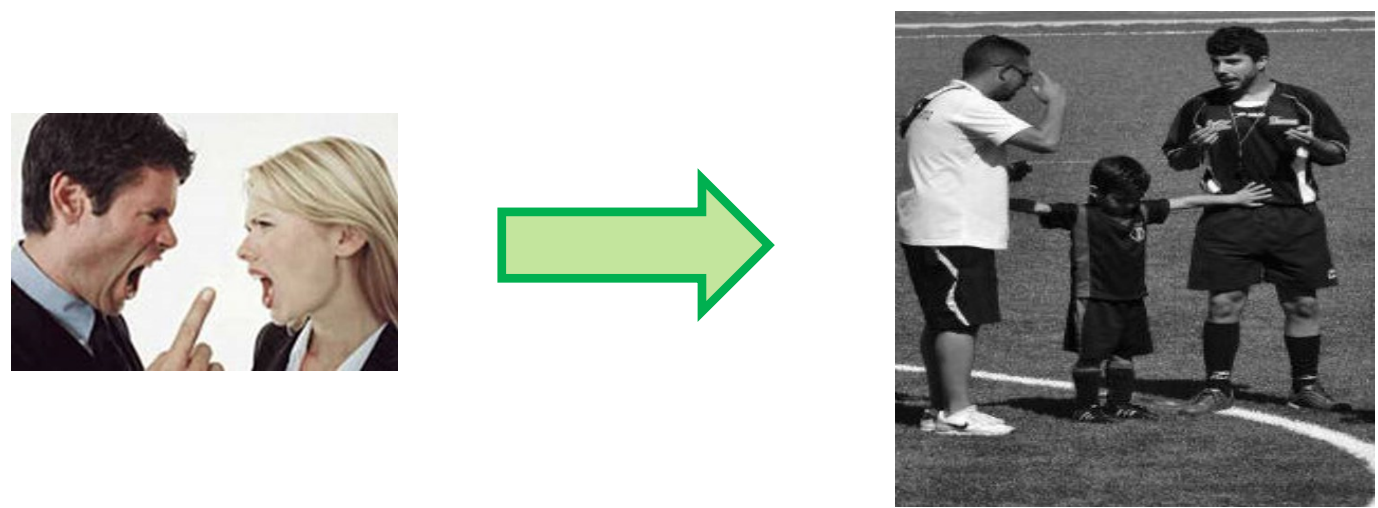

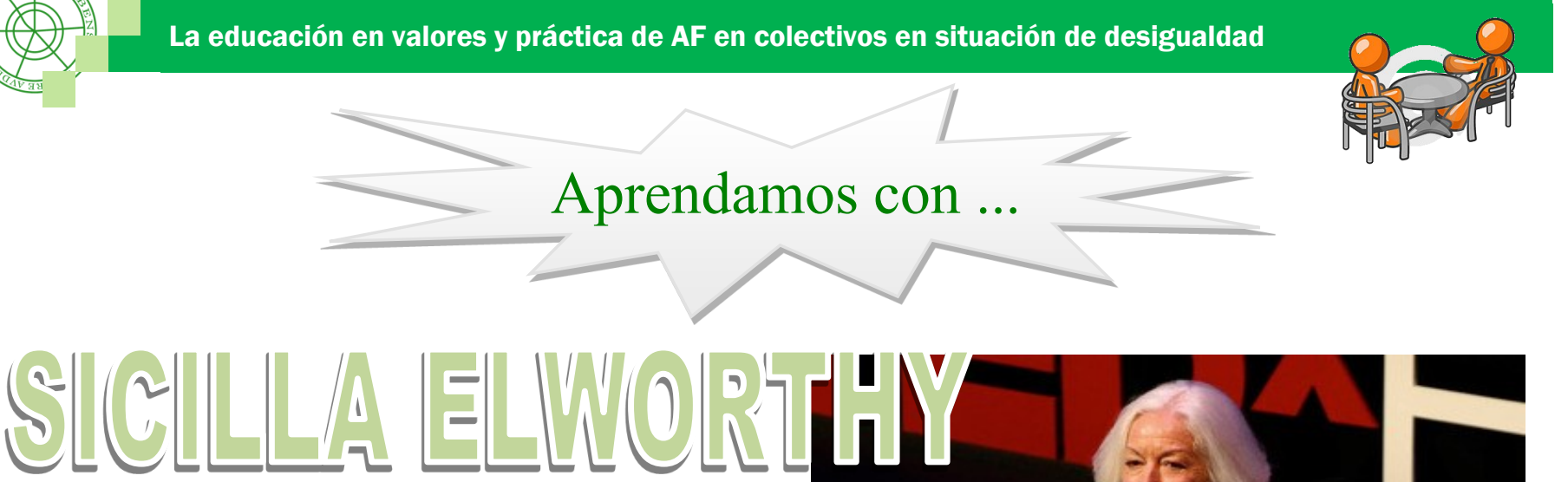

Elworthy, S. (2012).Fighting with non-violence. Ted. Revisado el

2 de diciembre, en http://www.ted.com/talks/ scilla_elworthy_fighting_with_non_violence/transcript?

language $=$ en $\#$ t-834296

Los militares británicos tienen un magnífico ejemplo que los inspira a la hora de la resolución de conflictos; es el brillante Teniente Coronel estadounidense llamado Chris Hughes. Él dirigía a

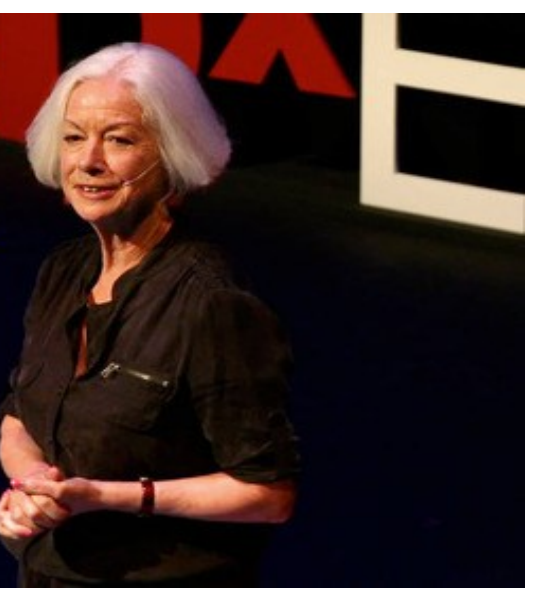
sus hombres por las calles de Nayaf — en Irak — y de repente la gente salió de sus casas y se amontonó a ambos lados de la calle, gritando, insultando, tremendamente enojados, y rodearon a estas tropas de jóvenes que estaban completamente aterrorizados, no sabían lo que estaba pasando y no hablaban árabe. Y Chris Hughes se abrió paso por en medio de la muchedumbre con el arma por encima de la cabeza, apuntando hacia el suelo, y les dijo: «Arrodíllense». Y estos soldados enormes con sus mochilas y trajes antibalas, tambalearon al suelo. Y todo quedó en completo silencio. Y después de 2 minutos, todos se dispersaron y se fueron a sus casas.

- Los agresores usan la violencia y solo en muy pocos casos, el recurrir a más violencia puede funcionar. Tenemos que darnos cuenta de que el uso de la fuerza contra la fuerza no funciona.

- La propuesta de Sicilla Elworthy pasa por usar el poder interior, a través del conocimiento de nosotros mismos, reconocer y trabajar con nuestro miedo, usar la ira como combustible, cooperar con los demás, hacer alianzas con otros, tener valor, y lo más importante, comprometerse activamente a la no violencia.

Link del video: $\mathrm{http}: / / w w w . t e d . c o m / t a l k s /$ scilla_elworthy_fighting_with_non_violence?language=es

\section{On-line}

\section{$\underline{\text { Redes- La mirada de Elsa- El inconsciente }}$}

Con este vídeo enlazamos el tema anterior del inconsciente con el tema tratado en este boletín, la resolución de conflictos. El inconsciente es una parte fundamental de nuestro cerebro que nos muestra cómo podemos llegar a comportarnos durante una situación problemática. Por ello debemos de aprender a utilizar la parte consciente de nuestro cerebro durante la resolución de conflictos.

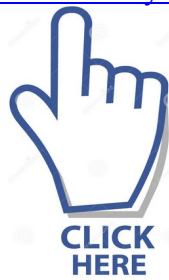




\section{Nuts illusion (The Jerry Andrus Version)}

En este video podemos observar como centramos la atención sobre los aspectos que nos interesan o sobre aquellos que nos inculca la sociedad, dejando sin ver otros muchos que pueden ser de vital importancia. Esto puede estar relacionado con el hecho de empatizar con la parte opuesta en un situación problemática.

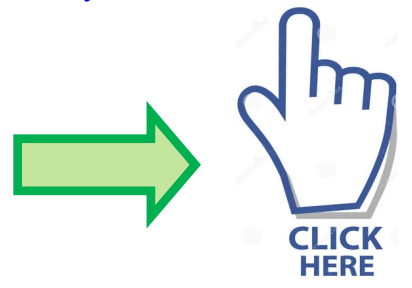

¿Por qué nos sacan de nuestras casillas algunos comportamientos de personas cercanas? à LEY DEL ESPEJO. Nos debemos preguntar: ¿Qué hay en mí que tanto me molesta de ti? Debe de haber algo que se escapa de tu propio control. Esto puede ser el detonante de que un comportamiento determinado te cause esa molestia.

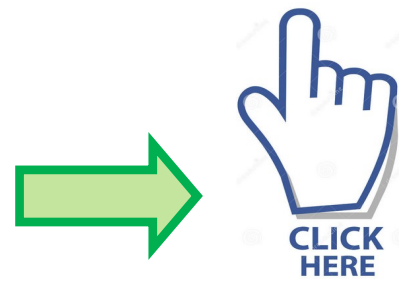

\section{Para Saber Más...}
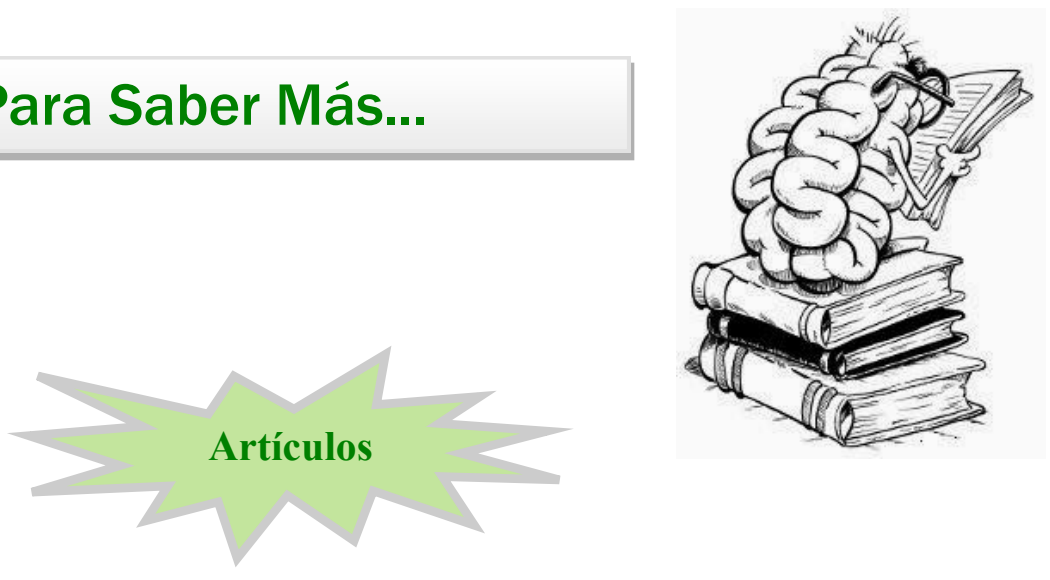

LA MEDIACIÓN EN LA RESOLUCIÓN DE CONFLICTOS

\section{Autor: Manuel de Armas Hernández}

El artículo presenta el desarrollo del proceso de mediación en la intervención del conflicto. En un primer apartado se define el concepto de mediación y sus características. Los apartados segundo y tercero recogen los aspectos relativos a las modalidades de mediación y su transformación ante el conflicto. Finalmente, se muestran las aportaciones de la mediación en el ámbito familiar, escolar y judicial entre otros. (De Armas Hernández, M. 2003).

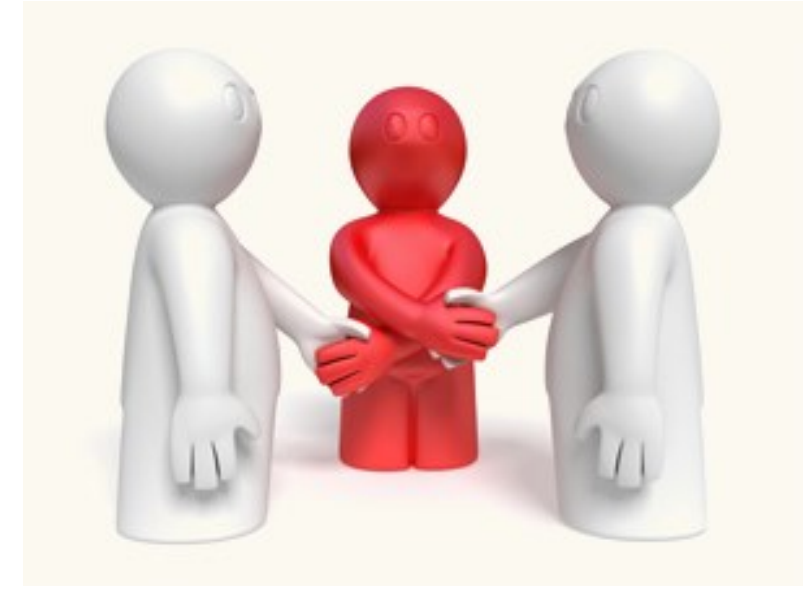




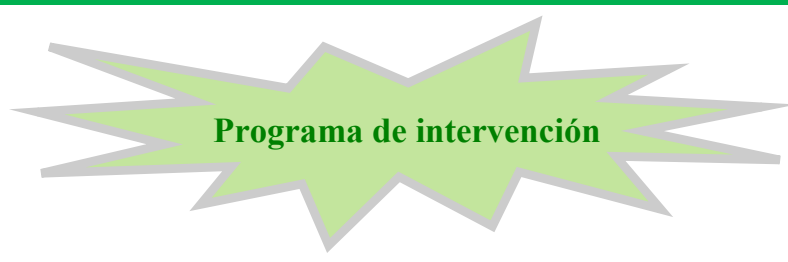

Programa de intervención para la resolución de conflictos estudiantiles dirigido a los jóvenes del séptimo $\left(7^{\circ}\right)$ grado de la UEN liceo "Andrés Bello". Una alternativa para la inducción de valores de la no-violencia hacia la convivencia ciudadana.

\section{Autora: María Elena Hidalgo}

La presente investigación tuvo como finalidad proponer un programa para la resolución de conflictos estudiantiles a través del diseño, para su posterior aplicación y evaluación, de un Taller para la Resolución de Conflictos, con la inducción de Valores de la No-violencia hacia la Con-

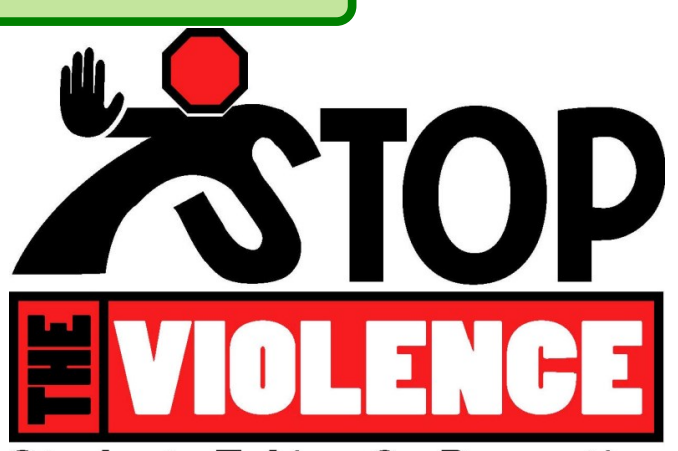
vivencia Ciudadana, en la Unidad Educativa Na-

cional "Andrés Bello", surgido de la apremiante necesidad de construir nuevas opciones ante la difícil situación de violencia estudiantil de dicha institución. El Taller para la resolución de Conflictos captó la atención de los involucrados, quienes evolucionaron paulatinamente de la irrupción social hacia la canalización de los conflictos. (Hidalgo, M. E., 2009).

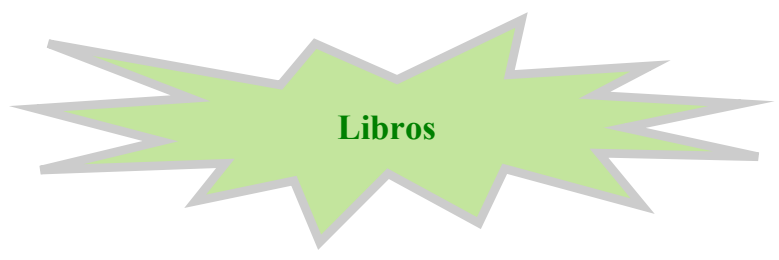

La resolución de los conflictos en y a través de la educación física.

Autores: Antonio Fraile Aranda, Jesús Vicente Ruíz, Carlos Velázquez y Víctor Manuel López Pastor

Este libro ofrece pautas cooperativas sobre cómo resolver conflictos que surgen en las clases, así como actividades y estrategias metodológicas que ayudan a los escolares a reflexionar sobre dichos comportamientos. Se tratan los conflictos en las clases de educación física y algunas estrategias para su resolución, educación física, valores éticos y resolución de conflictos: reflexiones y propuestas de acción y las actividades cooperativas como recurso para el tratamiento de los conflictos en las clases de educación física.

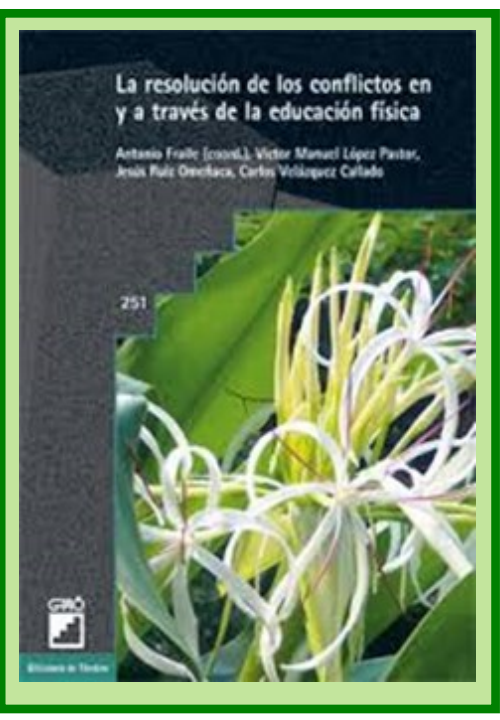


La ley del espejo.

\section{Autor: Yoshinori Noguchi}

A partir de una historia sencilla y emotiva, Yoshinori Noguchi (Hiroshima, 1963), un reconocido experto en coaching y asesoramiento psicológico, nos sitúa delante de un espejo para enfrentarnos con nuestro interior que es, en definitiva, el que determina todo lo que nos sucede en la vida. Eiko está preocupada porque los niños del colegio maltratan a su hijo Yuta, y se siente totalmente impotente y sola ante un problema que no sabe cómo resolver. Hasta que su amigo Yaguchi le ofrece un método sorprendente que la hará vivir la situación más difícil de su vida: enfrentarse a sus fantasmas y seguir adelante con espíritu nuevo. A medio camino entre el coaching y las constelaciones familiares, La ley del espejo nos propone pautas claras y efectivas para resolver de raíz los problemas de la vida.
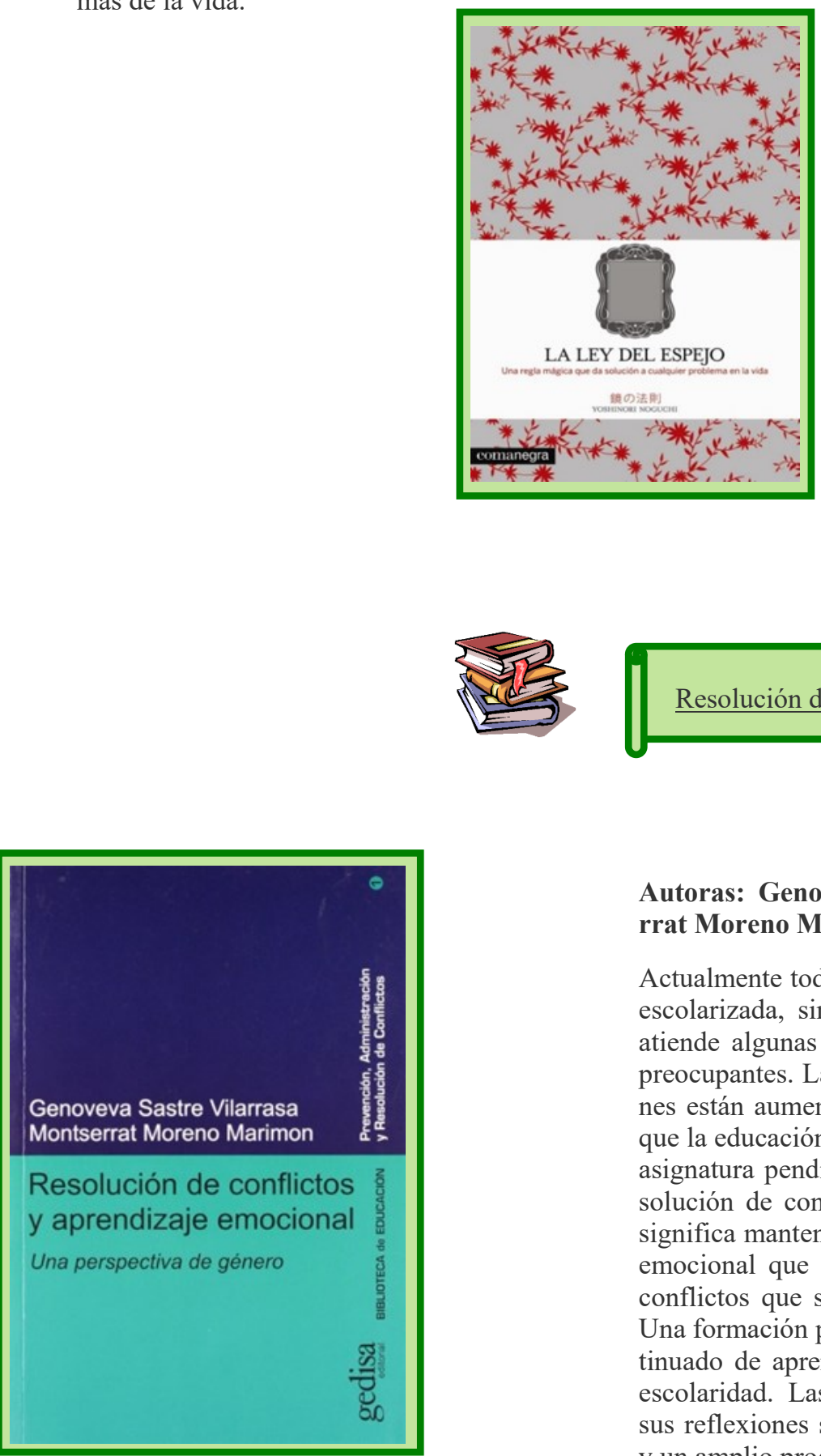

\section{Autoras: Genoveva Sastre Vilarrasa y Montse- rrat Moreno Marimon.}

Actualmente toda la población infantil española está escolarizada, sin embargo, la enseñanza actual no atiende algunas de las necesidades más urgentes y preocupantes. Las conductas violentas y las agresiones están aumentando entre los jóvenes, de manera que la educación emocional adecuada parece ser una asignatura pendiente. No tratar las emociones y resolución de conflictos en la enseñanza obligatoria, significa mantener al alumnado en un analfabetismo emocional que les dificultará la superación de los conflictos que se les presenten a todas las edades. Una formación para la vida requiere un proceso continuado de aprendizaje desde los primeros años de escolaridad. Las autoras ofrecen en este volumen sus reflexiones sobre esta enseñanza tan importante y un amplio programa de recursos y ejercicios. 


\section{Artículo de prensa.}

\section{MUERE JIMMY FRANCISCO ROMERO, EL AFICIONADO DEL DEPORTIVO, TRAS LA BATALLA}

CON ULTRAS DEL ATLÉTICO.

En la pelea han participado unas 200 personas, cuando a eso de las $09.00 \mathrm{~h}$. Los gallegos llegan en los autobuses a los alrededores de Madrid Río y se reúnen con los aficionados del Atlético de Madrid.

En la reyerta que tuvo lugar el día 30 de noviembre fallece el aficionado de los Riazor Blues. Este sufrió una parada cardiorrespiratoria y traumatismo craneoencefálico tras la lucha entre ultras, acabando en el río de donde lo rescataron un equipo de buceadores.

\section{LAPOLICÍA CIERRALA INVESTIGACIÓN POR LA MUERTE DEL ULTRA DEL DÉPOR.}

Cuatro presuntos autores materiales de la muerte de Francisco J. Romero Taboada en prisión, más de 80 detenidos y medidas para poner coto a los ultras en los estadios.

\section{INTERIOR APLICARÁ CONTRA LOS ULTRAS EL PLANQUEUSA PARA LASBANDAS LATINAS.}

El Ministerio del Interior ha incluido a los ultras de los equipos de fútbol en su Plan de actuación contra grupos organizados y violentos de carácter juvenil.

Link del vídeo: https://www.youtube.com/watch?v=J_-LcR9tMm8

\section{REFLEXIÓN DEL VÍDEO.}

Lo ideal en dicho conflicto habría sido que ambas partes asumieran sus responsabilidades en dicho problema (muerte de Francisco Javier Romero Taboada) en vez de acusarse mutuamente. Esto ha provocado que aumente la rivalidad y tensiones entre ambos grupos.
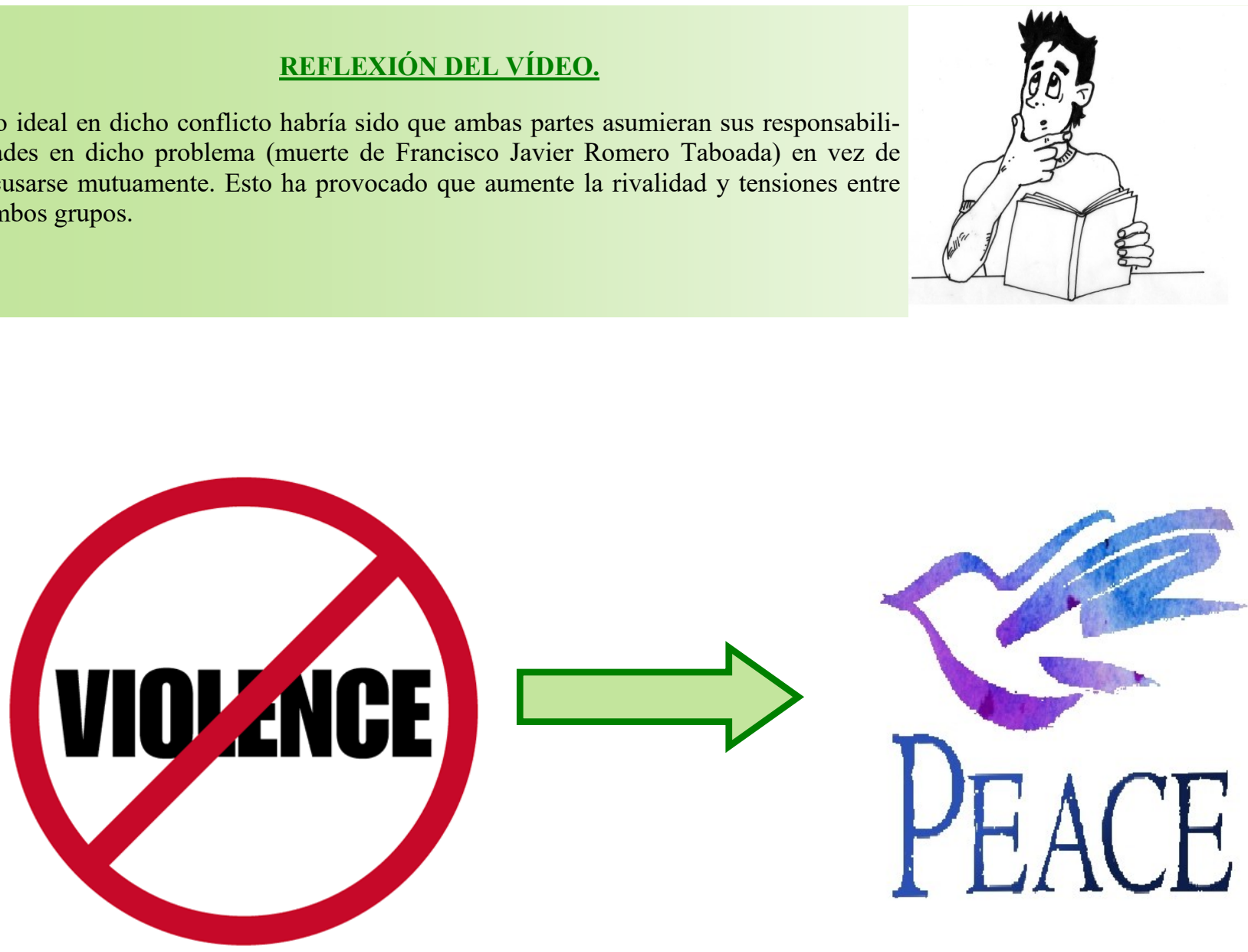


\section{Conclusiones.}

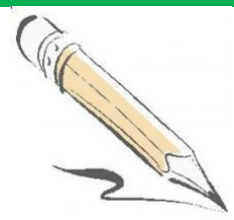

La formación de profesores de educación física, monitores deportivos, entrenadores, etc. en cuanto a la resolución de conflictos debe ser bastante completa, ya que ellos son los que se enfrentan habitualmente a situaciones problemáticas, las cuales son más propensas a darse en contextos de competición deportiva. De esta manera conocerán el procedimiento de actuación y a la misma vez ofrecerán una lección a las partes integrantes del conflicto.

Así mismo, la formación de estos profesionales será de vital importancia para que los alumnos reciban una educación emocional lo suficientemente rica, de modo que, le permita afrontar y superar en sus vidas aquellos conflictos que se le presenten.

Por último, creemos que un factor esencial en la resolución de conflictos es el hecho que las partes del conflicto empaticen con sus opuestos. En la resolución del conflicto es importante que este se afronte con tranquilidad, lo que nos permitirá una mejor resolución del problema, rehusando el recurso de la violencia..

\section{Sáenz-López, P. (2014). Apuntes inéditos de la asignatura “La educación en}

\section{Bibliografía.}

$\diamond \quad$ Artículos:

- De Armas Hernández, M. (2003). La mediación en la resolución de conflictos. Universitat de Barcelona. Departament MIDE.

- Hellison, D. (1978). El Modelo de Enseñanza de la Responsabilidad Personal y Social a través del Deporte. Revista de Educación 341.

- Hidalgo, M. E. (2009). Programa de intervención para la resolución de conflictos estudiantiles dirigido a los jóvenes del séptimo $\left(7^{\circ}\right)$ grado de la UEN Liceo. Dialnet. http://dialnet.unirioja.es/servlet/articulo?codigo=3175962

- $\quad$ Ruiz, L. M; Rodríguez, P; Martinek, T; Schilling, T; Durán, L. J; Jiménez, P. (2005). El proyecto Esfuerzo: Un modelo para el desarrollo de la responsabilidad personal y social a través del deporte. Revista de educación 341. 
$\diamond \quad$ Enlaces on-line y websites:

- $\quad$ Descubre la Ley del Espejo para ser Feliz. Mejor con salud. http://mejorconsalud.com/descubre-la-leydel-espejo-para-ser-feliz/

- $\quad$ Elworthy, S. (2012).Fighting with non-violence. Ted. http://www.ted.com/talks/ scilla elworthy fighting with non violence/transcript?language=en\#t-834296

- $\quad$ González, A. (2014). Normopatía. Centinela 66. Revisado el 2 de Diciembre de 2014 en, https:// centinela66.wordpress.com/tag/normopatia/

- $\quad$ Normopatía. (30 de Mayo 2012). Cyclopaedia. Revisado el 2 de Diciembre de 2014 en, http:// es.wikipedia.org/wiki/Normopat $\% \mathrm{C} 3 \% \mathrm{ADa}$

- Para, E. (2014). La policía cierra la investigación por la muerte del ultra del Dépor. El país. Deportes. http:// deportes.elpais.com/deportes/2014/12/30/actualidad/1419946222 536851.html

- $\quad$ Ramírez, D. (2008). El principio 90/10. Slideshare. Revisado el 2 de Diciembre de 2014, en http:// es.slideshare.net/davinken/el-principio-90-10-presentation

- Venegas, L. (2011). Normopatía. Psicólogos Perú. Revisado el 2 de Diciembre de 2014 en, http:// psicologosperu.blogspot.com.es/2011/08/normopatia.html

$\diamond \quad$ Libros:

- $\quad$ Fraile Aranda, A; Ruíz, J.V; Velázquez, C; López Pastor, V. M. (2008). La resolución de los conflictos en y at través de la educación física. GRAO.

- $\quad$ Noguchi, Y. (2010). La ley del Espejo. Comanegra.

- $\quad$ Sastre Vilarrasa, G; Moreno Marimon, M. (2002). Resolución de conflictos y aprendizaje emocional. Gedisa.

$\diamond \quad$ Apuntes:

- $\quad$ Sáenz-López, P. (2014). Apuntes inéditos de la asignatura "La educación en valores y la práctica de actividad física en colectivos en situación de desigualdad". Grado de Ciencias del Deporte. Universidad de Huelva. 